\title{
Symptome unterscheiden - Allergie oder Atemwegsinfekt?
}

Der Pollenflug der Birke ist heuer besonders intensiv. Weil er außerdem mit dem Auftreten des Coronavirus SARS-CoV-2 zusammenfällt und die allergischen Beschwerden denen einer viralen Atemwegsinfektion ähneln, ist die genaue Unterscheidung umso wichtiger.

Der Winter 2019/2020 war ungewöhnlich mild - der zweitwärmste der Messgeschichte laut ZAMG (Zentralanstalt für Meteorologie und Geodynamik). Die Pollensaison startete daher heuer mit der Blüte von Hasel und Erle vor allem im Westen Österreichs um sieben bis zehn Tage früher und auch deutlich stärker als im langjährigen Schnitt. Dazu bescherte das ständige Auf und Ab der Temperaturen im März Allergikern einen Wechsel an belastungsintensiven und -freien Tagen. Nach dem Abblühen von Hasel und Erle folgte die nächste Belastungswelle mit den Eschenpollen, deren allergologisches Potenzial oft unterschätzt wird.

\section{Starke Birkenblüte}

Sobald die Temperatur konstant auf über 15 Grad Celsius klettert, beginnt die Birke ihren Pollen an den Wind abzugeben (• Abb. 1). „Die Pollenmenge wird heuer deutlich über dem langjährigen Schnitt liegen - sogar noch über jener des Vorjahres, die ohnehin schon hoch war", informiert Mag. Maximilian Bastl vom Österreichischen Pollenwarndienst der MedUni Wien. Damit wird der beliebte Alleebaum sein biologisches Muster (einer schwächeren Saison folgt eine starke) unterbrechen.

\section{Plötzlicher Blühbeginn stresst Pflanze und Immunsystem}

Die Birke ist als häufiges und starkes Allergen bekannt. Etwa die Hälfte aller Allergiker reagiert auch auf Birkenpollen (- Abb. 2). Neben der Pollenmenge hat auch der Verlauf der Saison wesentlichen Einfluss auf die empfundene Belastung.

ע) Steigt die Pollenkonzentration langsam an, kann sich der Körper besser auf die Belastung einstellen

Steigt die Pollenkonzentration langsam an, kann sich der Körper besser auf die Belastung einstellen und empfindet die Saison als nicht so stark. Setzt der Pollenflug jedoch schlagartig ein, so wie heuer, wird die Saison stärker wahrgenommen.

) Auch die zunehmende Belastung durch Luftschadstoffe fordert ihren Tribut

Auch die zunehmende Belastung durch Luftschadstoffe fordert ihren Tribut. Denn durch Luftschafstoffe geraten Pflanzen unter Stress und bilden mehr Allergene. Um dem Einfluss der Luftqualität auf den Beschwerdezustand der Allergiker gerecht zu werden, wurde die Pollen App des Österreichischen Pollenwarndienstes überarbeitet und der AQI (AirQualitylndex) des Kooperationspartners Copernicus in die App aufgenommen.

\section{Pollenallergie oder virale Atem- wegserkrankung?}

Häufig werden allergische Symptome fälschlich als viraler Infekt missinterpretiert. „Aktuell verunsichert das besonders, da Viele fürchten, an COVID-19 (- Abb. 3) erkrankt zu sein", sagt Prof. Dr. Erika Jensen-Jarolim vom Institut für Pathophysiologie und All- ergieforschung an der MedUni Wien und Vizepräsidentin der Österreichischen Gesellschaft für Allergologie und Immunologie (ÖGAI). Die Expertin erklärt die wesentlichen Merkmale einer Allergie wie folgt (• Tab. 1).

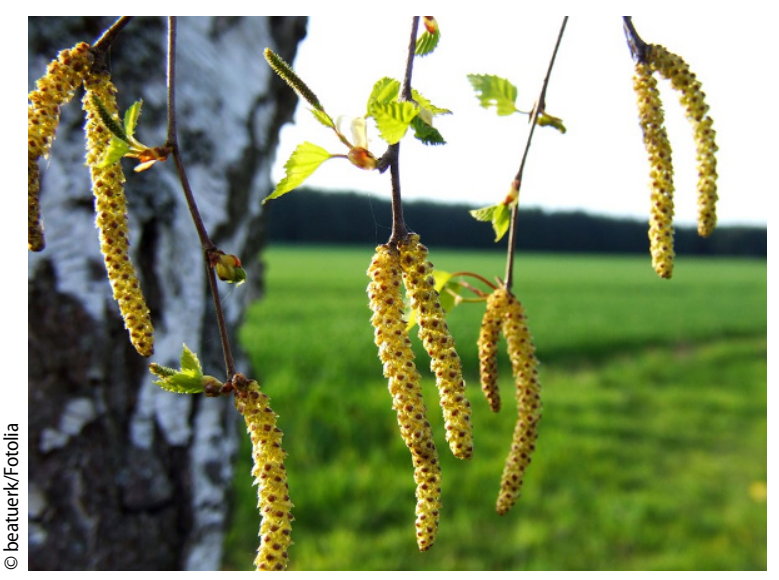

Abb. 1 \ Sobald die Temperatur konstant über 15 Grad Celsius steigt, fliegen die Birkenpollen

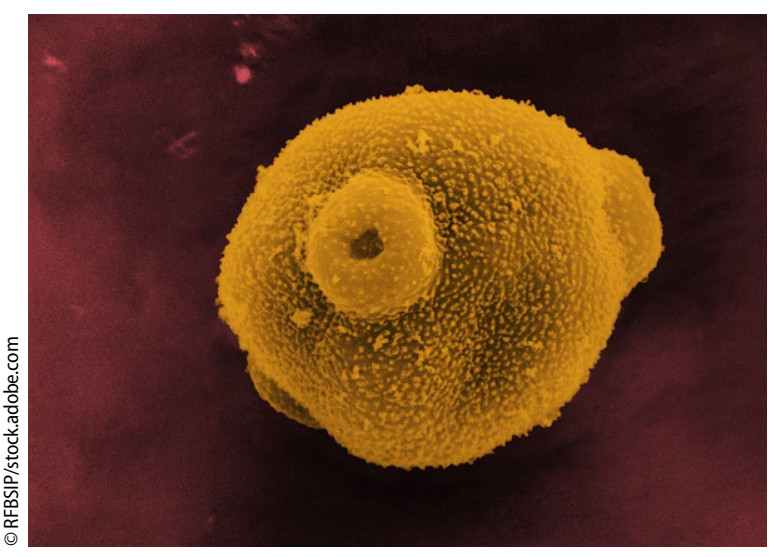

Abb. $2 \Delta$ Birkenpollen unter dem Elektronenmikroskop 
Tab. 1 Symptome einer Pollenallergie und einer Coronavirus-Infektion im Vergleich

\begin{tabular}{|c|c|c|}
\hline & Pollenallergie & COVID-19 (Coronavirus)* \\
\hline Zeitpunkt/Beginn & $\begin{array}{l}\text { Tritt jedes Jahr etwa zur gleichen Zeit auf: Birkenpol- } \\
\text { lenallergie Ende März, Gräserpollenallergie Anfang/ } \\
\text { Mitte Mai }\end{array}$ & Plötzlicher Krankheitsbeginn \\
\hline Verlauf & Ändert sich während der Pollensaison immer wieder & $\begin{array}{l}80 \% \text { der Fälle verlaufen mild bis moderat. In schwe- } \\
\text { ren Fällen ist eine rasche Verschlechterung möglich }\end{array}$ \\
\hline $\begin{array}{l}\text { Symptome an Augen } \\
\text { und Nase }\end{array}$ & $\begin{array}{l}\text { Beschwerden sind in erster Linie hier spürbar: } \\
\text { Die Augen jucken und sind oftmals - durch eine } \\
\text { Entzündung der Bindehaut - gerötet. Die Nase } \\
\text { juckt oft ebenfalls und läuft permanent oder ist } \\
\text { verstopft. Es kann zu Niesanfällen kommen, sobald } \\
\text { man einer vermehrten Pollenbelastung ausgesetzt } \\
\text { ist (der Österreichische Pollenwarndienst informiert } \\
\text { stundenaktuell) }\end{array}$ & Keine Beschwerden an Augen und Nase \\
\hline Fieber & Nein & Ja, ist ein Leitsymptom \\
\hline Atemwege & $\begin{array}{l}\text { Durch die allergiebedingt verengten und verschleim- } \\
\text { ten Bronchien kann es zu Atemnot (bis Asthma) und } \\
\text { pfeifenden Atemgeräuschen („Giemen“) besonders } \\
\text { beim Ausatmen kommen }\end{array}$ & $\begin{array}{l}\text { Starker trockener Husten \& Kurzatmigkeit } \\
\text { In sehr schweren Fällen Lungenentzündung, schwe- } \\
\text { res akutes Atemwegssyndrom oder Lungenversagen }\end{array}$ \\
\hline Allgemeinbefinden & $\begin{array}{l}\text { Abgeschlagenheit und Müdigkeit sind häufige } \\
\text { Folgen von schlechtem Schlaf: Durch ungenügende } \\
\text { Nasenbelüftung nach Allergenkontakt besteht } \\
\text { Sauerstoffmangel der roten Blutkörperchen }\end{array}$ & $\begin{array}{l}\text { Müdigkeit, Abgeschlagenheit im Rahmen der } \\
\text { Allgemeinsymptome bei viralem Infekt, mitunter } \\
\text { Schmerzen von Muskeln, Gelenken, Hals und Kopf. } \\
\text { Manchmal Übelkeit/Erbrechen und Durchfall }\end{array}$ \\
\hline $\begin{array}{l}\text { Behandlung der } \\
\text { Symptome }\end{array}$ & $\begin{array}{l}\text { Die Beschwerden verbessern sich bei Einnahme bzw. } \\
\text { Verabreichung Symptom-lindernder Medikamente } \\
\text { wie Antihistaminika und Kortison }\end{array}$ & $\begin{array}{l}\text { Symptomatische Behandlung ( } z \text {. B. fiebersenkende } \\
\text { Medikamente - vorrangig Paracetamol) und Bettru- } \\
\text { he. Bei schwereren Fällen } 1450 \text { anrufen }\end{array}$ \\
\hline $\begin{array}{l}\text { Ursächliche Behand- } \\
\text { lung }\end{array}$ & $\begin{array}{l}\text { Allergen-spezifische Immuntherapie: seit vielen } \\
\text { Jahre bewährt \& nachhaltig wirksam }\end{array}$ & Noch keine ursächliche Therapie verfügbar \\
\hline Prävention & $\begin{array}{l}\text { Allergenvermeidung (z. B. Pollenfilter, Vermeidung } \\
\text { von Outdoor-Aktivitäten abhängig vom Pollenflug, } \\
\text { Aufenthalte am Meer) }\end{array}$ & $\begin{array}{l}\text { Infektionsprophylaxe (durch Hygiene-Maßnahmen, } \\
\text { Einschränkung sozialer Kontakte und Reisetätigkeit) }\end{array}$ \\
\hline
\end{tabular}

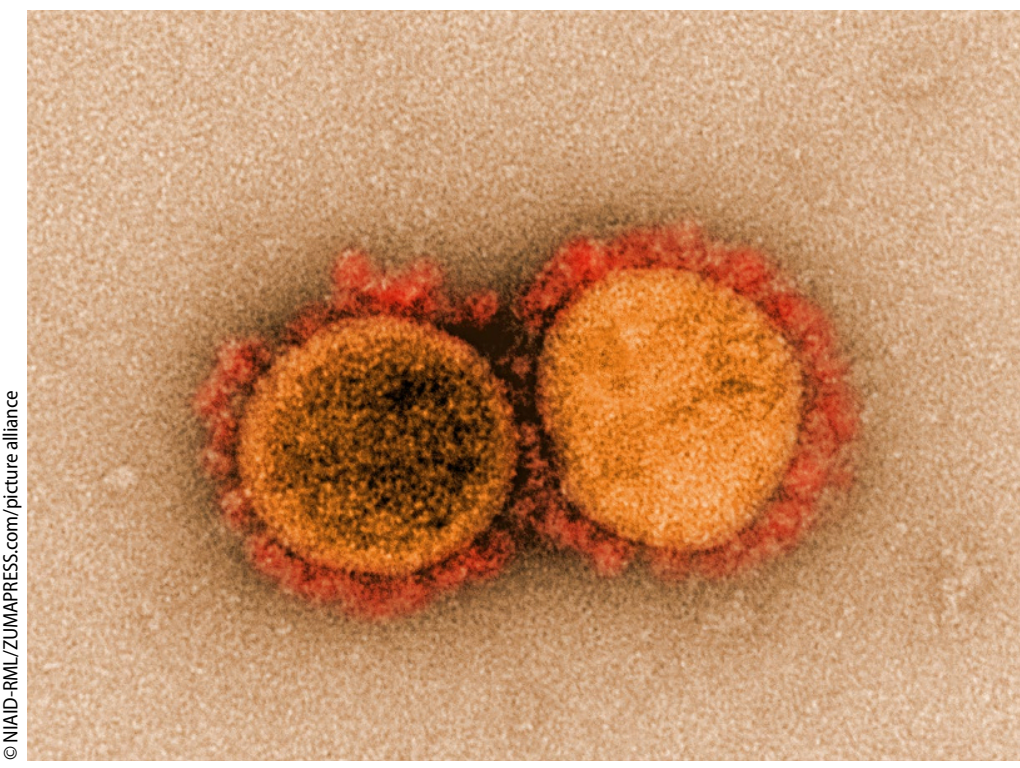

Abb. $3 \triangle$ SARS-CoV-2

\section{Weitere Informationen}

www.pollenwarndienst.at www.allergenvermeidung.org www.ages.at/themen/krankheitserreger/ coronavirus

Hinweis des Verlags. Der Verlag bleibt in Hinblick auf geografische Zuordnungen und Gebietsbezeichnungen in veröffentlichten Karten und Institutsadressen neutral.

hautnah $2020 \cdot 19: 45-47$

https://doi.org/10.1007/s12326-02000376-9

(c) Springer-Verlag GmbH Austria, ein Teil von Springer Nature 2020 
Hier steht eine Anzeige.

Springer 